\title{
Caregiving Stress Among American Indians With Type 2 Diabetes: The Importance of Awareness of Connectedness and Family Support
}

Journal of Family Nursing 2018, Vol. 24(4) 62I-639

(C) The Author(s) 2018

Article reuse guidelines: sagepub.com/journals-permissions DOI: $10.1177 / 10748407 / 8810550$ journals.sagepub.com/home/jfn

@SAGE

\section{Ashleigh Coser, MS', Kelley J. Sittner, PhD', Melissa L. Walls, PhD $^{2}$, and Tina Handeland, $\mathbf{M A}^{3}$}

\begin{abstract}
American Indian (Al) communities experience a disproportionate rate of Type 2 diabetes (T2D) and cumulative exposure to stress. Although this link is well researched among various populations, it has not been examined among Al communities. Path analysis was used to examine a multiple-mediator model to explain how caregiver stress influences self-reported mental and physical health among 100 Al participants with T2D. Caregiver stress was negatively associated with physical and mental health. Physical health was positively associated with family/community connectedness and mental health was positively associated with both family support and connectedness. The relationship between caregiver stress and mental health was partially mediated by family/community connectedness; caregiver stress had no indirect effects on physical health via either hypothesized mediator. Findings demonstrate the importance of integrating individuals' connection to family and community and its influence on caregiver stress and mental health in intervention programs targeting diabetes management and care among $\mathrm{Al}$ communities.
\end{abstract}

\footnotetext{
'Oklahoma State University, Stillwater, USA

2University of Minnesota Medical School, Duluth, , USA

IIndependent Scholar, WI, USA
}

\section{Corresponding Author:}

Ashleigh Coser, Department of Psychology, Oklahoma State University, I06 N. Murray,

Stillwater, OK 74078, USA.

Email: acoser@okstate.edu 


\section{Keywords}

American Indians, Type 2 diabetes, stress, family, community

Psychosocial stressors are widely cited as fundamental agents of worsened health and reduced quality of life (Fisher, Chesla, Mullan, Skaff, \& Kanter, 2001; Krieger, 2014; Naranjo, Hessler, Deol, \& Chesla, 2012; Pearlin, Menaghan, Lieberman, \& Mullan, 1981; Szanton, Gill, \& Allen, 2005; Walters, Simoni, \& Evans-Campbell, 2002). The linkages between stress and well-being are particularly salient for individuals diagnosed with Type 2 diabetes (T2D) in that stress is a known etiological factor for diabetes onset and influences disease progression (Jiang et al., 2008; Walders-Abramson et al., 2014). Thus, there is impetus to identify specific domains of stress related to diabetes outcomes as well as coping resources that may mediate the impact of stress on health. This is particularly true for many minority communities where cumulative exposure to stress and disproportionate rates of T2D converge, especially among tribal communities (Krieger, 2001; Martin, Yurkovich, \& Anderson, 2016; Scarton, Bakas, Miller, Poe, \& Huber, 2014; Tashiro, 2005; Tiedt \& Brown, 2014). Recent estimates indicate that American Indian (AI) and Alaska Native rates of T2D are over double that found in the United States overall (Centers for Disease Control and Prevention [CDC], 2016), and diabetes is a leading cause of death for AI communities (Xu, Murphy, Kochanek, \& Bastian, 2016).

The social contexts of stress and diabetes may be especially influential in care and disease management. For example, socially supportive networks may encourage healthy behavior, reduce stress, and reduce disease burden (Bonds, Gondoli, Sturge-Apple, \& Salem, 2002; DiMatteo, 2004; Gallant, 2003; Naranjo et al., 2012; Scarton et al., 2016; van Dam et al., 2005). As a result, it is important to consider individuals with T2D as part of a larger system including the family, as these networks may further affect their health and well-being. These effects may be amplified for AIs as families and tribal communities serve as a major source of support (Goins, Noonan, Gonzales, Winchester, \& Bradley, 2017; Hill, 2006; Naranjo et al., 2012; Walters et al., 2002).

The Indigenous Stress-Coping Model (ISCM; Walters et al., 2002) identifies cultural practices and the inclusion of family and community as protective factors that can buffer or mediate negative mental and emotional outcomes in the face of stress and trauma. Numerous AI cultural factors including positive ethnic identity, involvement in cultural activities, and spirituality have been previously linked to positive mental and behavioral health outcomes (Carlson et al., 2017; Kulis, Hodge, Ayers, Brown, \& Marsiglia, 2012; LaFromboise, Hoyt, Oliver, \& Whitbeck, 2006; Schiefer \& Krahé, 
2014). Furthermore, both family and community are a direct tie to the continuation and practice of AI culture (Walters et al., 2002). Family structure among AIs is broadly defined in the sense that families commonly include both nuclear and extended family members (Garrett et al., 2014; Jones \& Lindahl, 2011). This is in contrast to the "traditional" nuclear family structure typically found among the majority culture. In addition to extended family members, community members and elders who are close with the family are often acknowledged and considered family (LaFromboise \& Dizon, 2003). Benefits of being connected to multiple generations and having an extended kinship network may provide more opportunities for social support and the intergenerational transmission of cultural practices (e.g., Jones \& Lindahl, 2011). Such relevance is highlighted in a parenting curriculum utilized in some of the communities involved in this study: "Caring for one another begins with our family and extends to the general community. The interdependence of the family support system that strengthened Anishinaabe in all manners of living" (Smart, St. Germaine, and Contributors, 1989). Hill (2006) described this as connectedness and it reflecting the individuals' sense of belonging to family and the community, and being invested in the wellbeing of those systems. Moreover, participants in Martin and colleagues (2016) study identified a connection with family and community as a significant asset to individuals' well-being and care of T2D.

Although previous work has discussed the role of the family and connectedness in reducing stress in AI families (Hill, 2006; Martin \& Yurkovich, 2014; Scarton et al., 2016), limited research exists examining stress and coping of caregivers with T2D. To date, research among AI communities has consisted of primarily examining barriers to treatment (Martin et al., 2016; Scarton et al., 2014) and the stress experienced by those providing care of individuals with T2D (e.g., Naranjo et al., 2012). Narratives from community members involved in the current study shared stories reflecting struggles with caregiving while maintaining one's own health:

My kids are diabetic. My son and my daughter are both diabetic. They were diabetic before me. So I kind of felt guilty because they were diabetic. I was thinking, what did I do wrong? What did I cook? You know? And then, so now they are taking care of themselves. But. . I get worried about them more than I get worried about my diabetes. I worry about them: "take your medicine" "go see your doctor"- and then they get mad at me because they go, "you're not even taking care of yourself!" (Female)

Another community member describes a similar experience as a caregiver for other family members: 
It gives me a lot of stress to try to be the caretaker of the family. That's pretty stressful. And both my parents are diabetic so I'm a part of a diabetic family and that stresses me out too. I try to do the best I can to stay healthy. (Male)

These narratives help to demonstrate that while caregiving and family can be protective, it can also be stressful and influence physical and mental health. Therefore, exploring caregiving as a potential stressor for individuals with T2D may have broader implications for their own disease management. In consequence, the current study utilized a culturally informed theoretical framework for understanding stress and coping among AI caregivers with T2D.

\section{Purpose of the Study}

Using the ISCM, the study sought to extend the model to explore connection to family and community as an underlying mechanism for the link between caregiver stress and self-reported health for AI participants. The purpose of this study was twofold: first, to examine associations between caregiver stress and physical and mental health, and second, to determine possible protective associations between family, community, and health. Following the ISCM, we hypothesized the following:

Hypothesis 1 (H1): Caregiver (parenting) stress will be negatively associated with self-rated physical and mental health.

Hypothesis 2 (H2): Family support and awareness of connectedness (a culturally meaningful indicator of family and communal connection) will be positively related to mental and physical health.

Hypothesis 3 (H3): Family support and awareness of connectedness will mediate the association between caregiver stress and physical and mental health.

\section{Method}

The Maawaji' idi-oog Mino-ayaawin (Gathering for Health) project is a community-based participatory research (CBPR) collaboration between a Midwestern university and five AI communities. Tribal resolutions supporting the project were granted by all five tribal nation governments prior to submission of the application for research funding. Community research councils (CRCs) on each reservation worked in close collaboration with the university-based research team to develop, refine, and implement study procedures and instruments. Final methodology was reviewed and approved by the University of Minnesota's institutional review board (IRB) and the Indian Health Service National IRB. 
The study involved two major phases: (a) a qualitative step including two sets of focus groups to identify salient community stressors and adapt survey measures (from which earlier referenced community quotations were drawn) and (b) a quantitative phase including survey data from computer-assisted personal interviews (CAPIs). The goal of the two-part process was to maximize measurement validity for local culture and contexts. The research team piloted any adapted measures with a convenience sample of Native adults. We further refined measures in collaboration with CRC members. During this process, we presented examples of survey questions and asked, for example, "does this measure seem to capture the idea of caregiver stress." Thus, validity is likely high for the measures included in this study. Caregiver stress questions were only asked of those participants who indicated that they were currently caring for dependent children. The terms caregiver and parent are used interchangeably in the current project.

\section{Participants}

To select participants for inclusion, clinic staff at each of the five partnering tribal health clinics generated probability (simple random) samples from tribal clinic records of individuals with a diagnosis of T2D, above the age of 18 years, living on or near the reservation, and self-identifying as AI. Selected recruits were mailed a study invitation letter and informational brochure and provided with mail and phone-in options for refusals. Trained community interviewers contacted nonrefusing recruits, answered questions about study procedures, and completed informed consent procedures for those interested in enrolling in the study. Survey interviews took place in private, safe spaces (e.g., participants' homes, local office spaces). The total baseline sample includes 194 participants (response rate $=67 \%$ ) who received a US $\$ 50$ incentive for completion of the CAPI between November 2013 and November 2015.

\section{Measurement}

Questions used to operationalize major measures included in these analyses are listed in the appendix section. All measures included in the study were reviewed and adapted based on feedback from focus groups, which consisted of community members involved in the study. Our focal independent variable, caregiver stress, was adapted from the Parental Stress Scale $(\alpha=$ .62; Berry \& Jones, 1995). Participants were asked first if they were currently caring for minor children; those who responded in the affirmative were asked whether they agreed or disagreed with seven statements measuring stress associated with being a caregiver. Response options ranged from $1=$ strongly agree to $4=$ strongly disagree. Statements 2, 3, 5, 6, and 
7 were reverse-coded. A mean score was calculated by averaging responses to the seven items. A higher value indicated more caregiver stress.

Two health outcomes, both from the World Health Organization's Composite International Diagnostic Interview (Wittchen \& Kessler, 1994), were included as dependent variables. Self-rated health measures have been shown to display exceptional predictive validity for mortality (Idler \& Benyamini, 1997). Despite inherent subjectivity, the validity of self-rated health appears to be increasing over time (Schnittker \& Bacak, 2014). Participants were asked to self-rate their overall physical health and mental health with a 5-point scale, ranging from $1=$ excellent to $5=$ poor. Responses were reverse-coded so that higher values indicate better health.

Two social context variables were hypothesized to mediate the relationship between caregiver stress and the self-rated health outcomes. The first, family support, was adapted from Shields, Franks, Harp, Campbell, \& McDaniel's (1994) Family Emotional Involvement and Criticism Scale $(\alpha=.62)$. Participants were asked how strongly they agreed or disagreed with four items that assessed participants' perceptions of approval and support from family. Response options ranged from 1 = strongly agree to 4 = strongly disagree. As another measure of context, participant's awareness of connectedness was adapted from Mohatt, Fok, Burket, Henry, and Allen (2011) and assessed the degree to which participants feel connected to family and community $(\alpha=.68)$. Participants used a 3 -point scale $(1=$ not at all; $2=$ somewhat; $3=$ a lot $)$ in response to four statements regarding their connectedness to family and community. Mean scores were calculated for both contextual variables. Higher values indicate more family support and a greater awareness of connectedness, respectively. Our measures appear to have adequate internal consistency based on recommended thresholds of .60 (Nunally, 1978). We also controlled for gender $(1=$ female; $0=$ male $)$ and reservation dwelling status $(1=$ on reservation; $0=$ off reservation $)$ in the path analysis.

\section{Analytic Strategy and Missing Data}

Analysis began with an examination of bivariate correlations among the study variables in SPSS 24. We then used MPlus 7.2 (Muthén \& Muthén, 2007 ) to conduct a path analysis (depicted in Figure 1) to test the direct relationship between caregiver stress and both health outcomes, as well as whether the social context variables mediated that relationship. The model had acceptable fit to the data, with comparative fit index (CFI) close to .95 and root mean square error approximation (RMSEA) close to $.06(\mathrm{Hu} \&$ Bentler, 1999). 


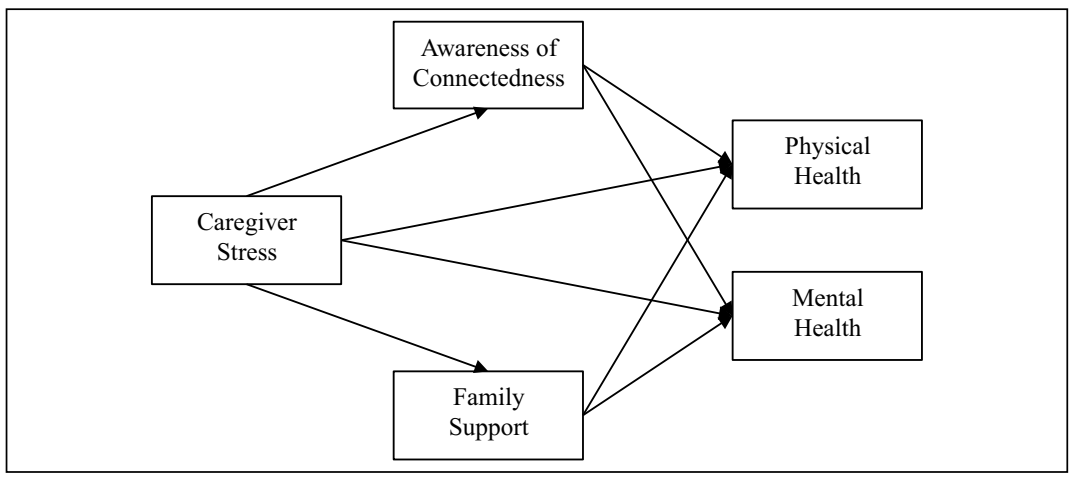

Figure I. Path model of caregiver stress and self-rated health. Note. Controlling for gender and on/off reservation status.

The current study utilized a subset of the original baseline sample, including only those participants who reported current caregiving status $(n=105$; $54.1 \%$ ). Five participants had missing data on the self-rated health outcomes, and there were no missing data for the other study variables. The analytic sample consisted of the 100 caregivers with data for self-rated physical and mental health. Path models utilized full information maximum likelihood estimation with robust standard errors (MLR), which is robust to no normality (Asparouhov \& Muthén, 2005; Muthén \& Muthén, 2007).

\section{Results}

Descriptive statistics for the variables used in the analyses are shown in Table 1. The subsample consisted of 61 women and 39 men. Most participants (83\%) lived on reservation land and $17 \%$ lived near reservation lands. Participants reported slightly better mental than physical health. Bivariate correlations are shown in Table 2. In support of our first hypothesis, caregiver stress was negatively associated with self-rated physical health and mental health $(r=-.25$, $p<.05$ for both). It was also negatively related to family support $(r=-.33, p<$ $.01)$ and to awareness of connectedness to family and community $(r=-.28$, $p<.01)$. Supporting the second hypothesis, family support and awareness of connectedness each had positive associations with self-rated mental health $(r=$ $.25, p<.05 ; r=.34, p<.01$, respectively). Only awareness of connectedness was positively associated with physical health $(r=.28, p<.01)$; the bivariate correlation for family support and physical health was not significant. The two social context variables were positively and significantly related to each other $(r=.20, p<.05)$. 
Table I. Descriptive Statistics $(n=100)$.

\begin{tabular}{lccccc}
\hline & M/\% & SD & Minimum & Maximum & $\alpha$ \\
\hline Physical health & 1.68 & 0.92 & 0 & 4 & \\
Mental health & 2.29 & 1.00 & 0 & 4 & \\
Caregiver stress & 1.18 & 0.40 & 0.29 & 2.71 & .62 \\
Family support & 1.94 & 0.42 & 0.75 & 3 & .62 \\
Awareness of connectedness & 1.27 & 0.44 & 0 & 2 & .68 \\
Female gender & $61.00 \%$ & & 0 & 1 & \\
On reservation & $83.00 \%$ & & 0 & 1 & \\
\hline
\end{tabular}

Table 2. Bivariate Correlations Among Study Variables $(n=100)$.

\begin{tabular}{|c|c|c|c|c|c|c|c|}
\hline & I & 2 & 3 & 4 & 5 & 6 & 7 \\
\hline I. Physical health & 1.00 & & & & & & \\
\hline 2. Mental health & $.44 * * *$ & 1.00 & & & & & \\
\hline 3. Caregiver stress & $-.25 *$ & $-.25 *$ & 1.00 & & & & \\
\hline 4. Family support & .15 & $.25 *$ & $-.33 * *$ & 1.00 & & & \\
\hline 5. Awareness of connectedness & $.28 * *$ & $.34 * *$ & $-.28 * *$ & $.20^{*}$ & 1.00 & & \\
\hline 6. Female gender & $-.21 *$ & $-.30 * *$ & .09 & .08 & -.14 & 1.00 & \\
\hline 7. On reservation & .07 & .98 & $-.21 *$ & .13 & .11 & -.03 & 1.00 \\
\hline
\end{tabular}

The standardized results from the path analysis are provided in Table 3. There were significant associations between caregiver stress and both social context variables. A standard deviation increase in caregiver stress was associated with a 0.33 standard deviation decrease in family support $(p<.01)$ and a 0.26 decrease in connectedness $(p<.01)$. As was the case for bivariate relationships, the two social context constructs had mixed relationships with the health outcomes. They were positively related to self-rated mental health but only connectedness was associated with self-rated physical health $(0.19$, $p<.10)$. There was no direct effect of caregiver stress on health, controlling for social context, gender, and living on a reservation.

To test our third hypothesis (i.e., whether social support and connectedness mediated the caregiver stress/health relationships), we examined the decomposition of effects for the path model (Table 4). Caregiver stress had no indirect effects on self-rated physical health via either awareness of connectedness or family support. Lending partial support to H3, the relationship between caregiver stress and mental health was partially mediated by awareness of 


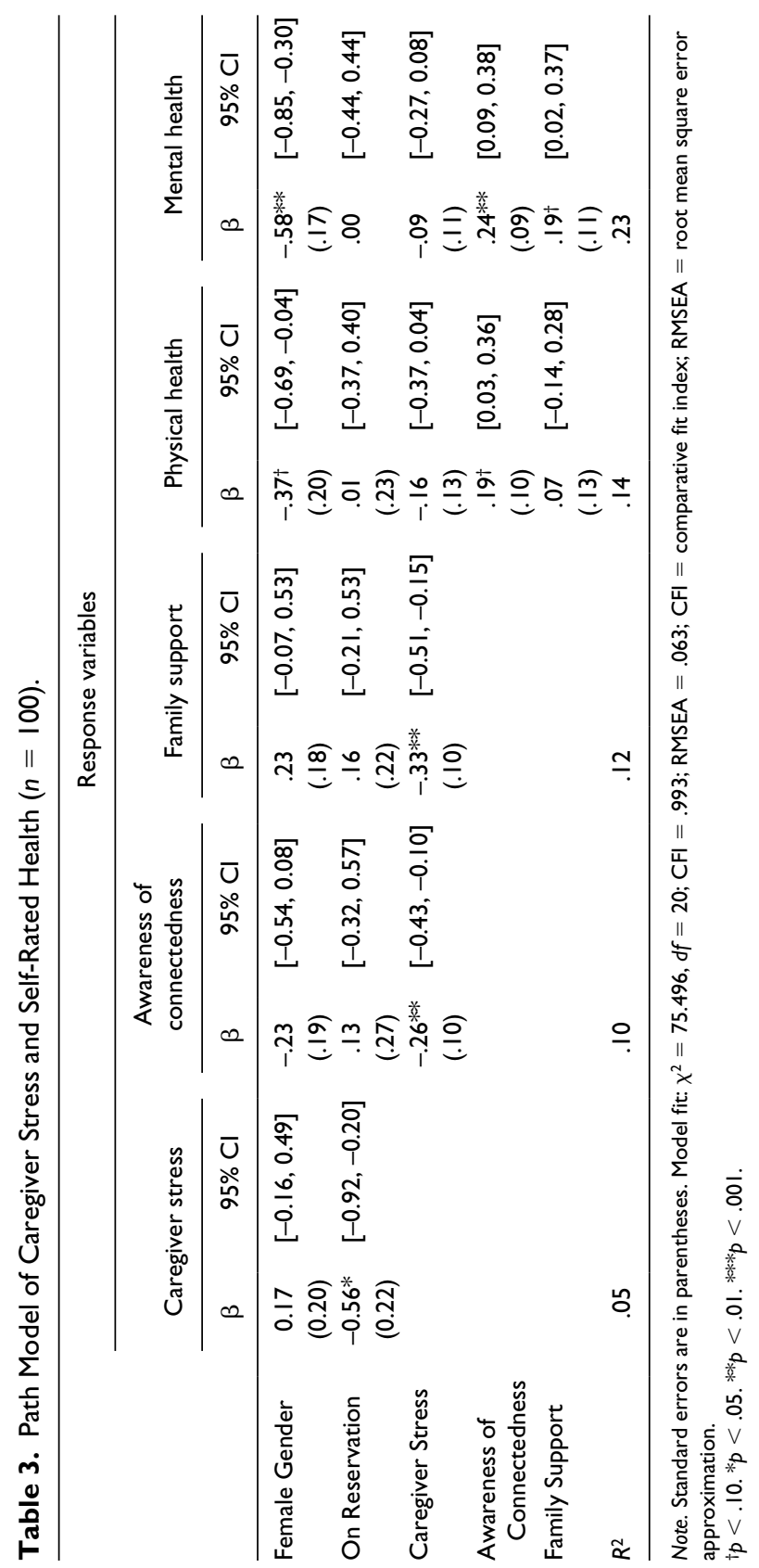


Table 4. Standardized Indirect and Total Effects of Caregiver Stress and Self-Rated Health.

Self-rated physical health Self-rated mental health

\section{Caregiver stress}

Indirect through

Family support

Awareness of connectedness

$-0.02$

$-0.06$

Total indirect effects

$-0.05$

$-0.06+$

$-0.07$

$-0.13^{*}$

$t_{p}<.10 . * p<.05$.

connectedness to family and community. The positive effect of connectedness on mental health was reduced by 0.06 standard deviations for each standard deviation increase in caregiver stress $(p=.07)$, with a total indirect effect of -0.13 standard deviations $(p<.05)$.

\section{Discussion}

The current project is a direct test of the ISCM (Walters et al., 2002) and lends support to this theory in that a culturally meaningful notion of connectedness offsets the impact of caregiver stress for mental health. The purposes of this study were first, to examine associations between caregiver stress and physical and mental health outcomes, and second, to examine possible protective effects of family support and awareness of connectedness (a culturally relevant construct) on these outcomes among a sample of AI caregivers living with T2D. The current study extends the ISCM by examining social and cultural factors as mediators of AI caregivers' stress and health.

The bivariate analyses demonstrated significant negative associations between caregiver stress and both health outcomes as well as both social context variables (i.e., family support and awareness of connectedness). This is important in the case of T2D given the role of stress (Jiang et al., 2008; Walders-Abramson et al., 2014) and social support (DiMatteo, 2004; Gallant, 2003; Goins et al., 2017) for diabetes management and prognosis. The current findings provide additional support that caregiver stress significantly and negatively affects the physical and mental well-being of patients with T2D. Furthermore, our findings build upon previous work by demonstrating associations between family support and an awareness of connectedness to others and reduced caregiver stress.

Bivariate results also reveal significant, positive associations between family support and awareness of connectedness and mental health. In addition, 
increased awareness of connectedness to family and community is linked with better self-reported physical health. Thus, while stressful contexts of child caregiving can be harmful to well-being, the presence of a supportive family and sense of connectedness can be advantageous for caregivers with T2D. Our findings are supported by previous literature discussing family and community connections as common cultural values for many AI people (Garrett et al., 2014; Jones \& Lindahl, 2011; LaFromboise \& Dizon, 2003; Martin et al., 2016), and ultimately, add to a growing body of literature documenting the potential protective impact of culturally meaningful constructs for AI health (e.g., Carlson et al., 2017; Hill, 2006; Kulis et al., 2012; LaFromboise et al., 2006; Martin et al., 2016; Schiefer \& Krahé, 2014).

Furthermore, our path analysis results demonstrate that awareness of connectedness to family and community partially mediates the link between caregiver stress and mental health. The ISCM (Walters et al., 2002) similarly discusses the importance of AI connections to family and community as positively affecting health in the face of stress. This mediating pathway is a direct empirical extension of ISCM and lends support to its theoretical framework in that a culturally meaningful notion of connectedness offsets the impact of caregiver stress for mental health. This sense of belonging and connectedness to the community has been linked with individuals' "health-promoting selfcare" (Hill, 2006, p. 213). Previous work has also discussed connectedness to family and community as protective against negative outcomes such as substance use and suicide (Mohatt et al., 2011).

An awareness of one's connectedness to family and community may represent a deeper construct with stronger cultural relevance and meaning than our basic measure of family support could capture. Indeed, the awareness of connectedness measure adapted for use in the current study was initially developed with AI communities in Alaska (Mohatt et al., 2011), while the family support measure was originally derived from work with primarily White adult patients (Shields et al., 1994). These considerations provide some explanation for our finding that family support did not mediate associations between caregiver stress and health. Scarton and colleagues (2016) discussed family support and caregiver needs as affected by cultural disparities in health care, lack of information and resources, managing the patient's emotions and behaviors, providing physical care, and providing instrumental care. These themes may ultimately help explain more of caregivers' stress and their well-being rather than general family support as measured in the current study. Future studies may include the needs identified by Scarton et al. (2016) as possible links to health among caregivers.

Although not hypothesized, we found that adults living on reservation lands reported lower levels of caregiver stress than those living off reservation. This 
finding may be due in part to the individuals' proximity and availability of resources and support provided by family and the community in smaller, closeknit reservation communities. Further investigation is needed to elucidate reasons for this potential protective effect of on-reservation living.

The results overall have implications for fostering family and community relationships and connections for AI people living with diabetes. This is especially true as these connections serve to not only positively affect mental health, but also may offset distress (Mohatt et al., 2011). Within a cultural context, the results reflect common practices among AI communities (Hill, 2006), which include the involvement of extended family/tribal community to support caregivers and children, and engagement in traditional activities (Walters et al., 2002). Connecting individuals and families to cultural programs providing traditional cultural activities is an example of how to foster relationships and connectedness to the community (Hill, 2006; Mohatt et al., 2011). This may also help to build a sense of belonging and social support. In addition, efficacy and reach of current interventions may be positively affected by including extended family members and/or targeting behavior change within the family as a unit rather than on an individual basis (Jones \& Lindahl, 2011; Martin \& Yurkovich, 2014).

\section{Limitations}

There are some limiting considerations when interpreting the current findings. These include methodological considerations, such as the cross-sectional study design, sample size, and analysis technique. The cross-sectional design precludes inferences of causation, and the small sample size means that we lack the statistical power to detect small effect sizes. Although path analysis with observed variables allows for smaller samples than models with latent variables, the use of observed variables does increase measurement error. In addition, study participants were seeking treatment at a reservation-based tribal health clinic. As a result, findings cannot be generalized to non-treatment-seeking individuals and those seeking treatment at a non-reservation-based clinics. Tribal and community representation may limit the generalizability of the findings to other AI populations, and restrict the exploration of possible ethnic and cultural differences. An additional consideration may include future exploration into operationalizing constructs, such as community connectedness, which appear to relate significantly to the health and well-being of AI populations. This examination may reveal conditions in which community connectedness is protective and ultimately provide necessary data to inform interventions. Furthermore, there is a need to examine utility of measures used in the current study to assess constructs such as caregiver stress and family support. Although 
all of the measures used in the current study were adapted and approved by project CRCs, there remains a desperate need for measurement development and psychometric work with AI communities. For example, it is difficult to assess how the shortened caregiver stress scale compares on internal consistency to the original 18-item scale because we have adapted it for use in a new culture and because Cronbach's alpha depends in part on the number of items included (Sijtsma, 2009).

\section{Conclusion}

The results of the current study are beneficial to AI communities and health providers, particularly for treatment considerations of adult caregivers living with T2D. The findings highlight the potential for enhancing coping resources to offset caregiver stress by way of family support and connection to family and community. Engaging AI family members in diabetes education and prevention has been shown to be feasible, acceptable, and impactful (Chambers et al., 2018), and coincides with evidence of AI preference for family support (relative to biomedical care) in help seeking (Aronson, Johnson-Jennings, Kading, Smith, \& Walls, 2016; Walls, Johnson, Whitbeck, \& Hoyt, 2006). Family- and community-involved diabetes treatment programs can be developed and evaluated using community-based participatory research approaches. As an example, the Together on Diabetes program was created in collaboration with the White Mountain Apache tribe and employs home-visiting health educators to deliver a structured curriculum; an interface with clinical providers including doctors, nurses, and diabetes nurse educators; and refer patients to community-based services and healthy activities (Chambers et al., 2015).

In sum, our findings support previous literature describing the role of family in health-maintenance behaviors and providing social support among AI caregivers with T2D. The link between caregivers' mental health and their sense of connectedness to family and community extends our knowledge of potential avenues to create and sustain a network of support for the individual and their health care. As such, the results of the current study support intergenerational models in which social connections are supported in culturally congruent, community-driven ways. In addition, our finding that caregiver stress is associated with worse health in this population of AI patients highlights the complexity of family dynamics: while families are known sources of support and resilience, the impact of family related stressors also deserves attention. Thus, family based interventions could be expanded upon to include and consider caregiving-related stressors. This may look like identifying existing social supports, enhancing and fostering ongoing relationships, and connecting patients and families with resources in the community. 


\section{Appendix}

Items Included in Scales.

Caregiver stress ( 1 = strongly agree; 2 = agree; 3 = disagree; $4=$ strongly disagree $)$

A. I am satisfied in my role as a caretaker. (reversed)

B. Caring for the children, I am responsible sometimes for taking more time and energy than I have to give.

C. I sometimes worry whether I am doing enough for the children that I care for.

D. The children whom I care for are an important source of affection for me. (reversed)

E. Having children to take care of has been a financial burden.

F. The behavior of the children that I care for is often embarrassing or stressful to me.

G. I feel overwhelmed by the responsibility of being a caretaker.

Family support ( $\mathrm{I}=$ strongly agree; 2 = agree; 3 = disagree; 4 = strongly disagree $)$

A. My family approves of the way I live my life. (reversed)

B. My family discourages me from talking about my difficulties.

C. My family does not like the way I take care of myself.

D. My family tries to get me to change.

Awareness of connectedness ( $1=$ not at all; $2=$ somewhat; $3=a$ lot $)$
A. When I am hurting, my family hurts with me.
B. My family's happiness is part of my happiness.
C. My community believes I am important.
D. My community's happiness is part of my happiness.

\section{Acknowledgments}

The authors gratefully acknowledge the Gathering for Health Community Research Council and Clinical Project Members: Sidnee Kellar, Rose Barber, Robert Miller, Tweed Shuman, Lorraine Smith, Sandy Zeznanski, Patty Subera, Tracy Martin, Geraldine Whiteman, Lisa Perry, Trisha Prentice, Alexis Mason, Charity Prentice-Pemberton, Kathy Dudley, Romona Nelson, Eileen Miller, Geraldine Brun, Murphy Thomas, Mary SikoraPetersen, GayeAnn Allen, Frances Whitfield, Phillip Chapman, Sr., Hope Williams, Betty Jo Graveen, Daniel Chapman, Jr., Doris Isham, Stan Day, Jane Villebrun, Beverly Steel, Muriel Deegan, Peggy Connor, Michael Connor, Ray E. Villebrun, Sr., Pam Hughes, Cindy McDougall, Melanie McMichael, Robert Thompson, and Sandra Kier.

\section{Declaration of Conflicting Interests}

The authors declared no potential conflicts of interest with respect to the research, authorship, and/or publication of this article.

\section{Funding}

The authors disclosed receipt of the following financial support for the research, authorship, and/or publication of this article: This project was supported by the National Institute of Diabetes and Digestive and Kidney Diseases (DK091250, M. Walls, PI). 


\section{References}

Aronson, B., Johnson-Jennings, M., Kading, M., Smith, R., \& Walls, M. (2016). Mental health service and provider preference among American Indians with type 2 diabetes. American Indian and Alaska Native Mental Health Research, 23, 1-23. https://doi.org/10.5820/aian.2301.2016.1

Asparouhov, T., \& Muthén, B. (2005). Multivariate statistical modeling with survey data. In Proceedings of the Federal Committee on Statistical Methodology (FCSM) research conference (pp. 14-16). Retrieved from https://www.statmodel .com/download/2005FCSM.pdf

Berry, J. O., \& Jones, W. H. (1995). The Parental Stress Scale: Initial psychometric evidence. Journal of Social and Personal Relationships, 12, 463-472. https://doi .org/10.1177/0265407595123009

Bonds, D., Gondoli, D., Sturge-Apple, M., \& Salem, L. (2002). Parenting stress as a mediator of the relation between parenting support and optimal parenting. Parenting: Science and Practice, 2, 409-435. https://doi.org/10.1207 /S15327922PAR0204_04

Carlson, A. E., Aronson, B. D., Unzen, M., Lewis, M., Benjamin, G. J., \& Walls, M. L. (2017). Apathy and type 2 diabetes among American Indians: Exploring the protective effects of traditional cultural involvement. Journal of Health Care for the Poor and Underserved, 28, 770-783. https://doi.org/10.1353/hpu .2017 .0073

Centers for Disease Control and Prevention. (2016). Summary health statistics: National Health Interview Survey: 2014 (Table A-4). Retrieved from http://ftp .cdc.gov/pub/Health_Statistics/NCHS/NHIS/SHS/2014_SHS_Table_A-4.pdf

Chambers, R. A., Rosenstock, S., Neault, N., Kenney, A., Richards, J., Begay, K., . . Barlow, A. (2015). A home-visiting diabetes prevention and management program for American Indian youth: The together on diabetes trial. The Diabetes Educator, 41, 729-747. https://doi.org/10.1177/0145721715608953

Chambers, R. A., Rosenstock, S., Walls, M., Kenney, A., Begay, M., Jackson, K., . . Barlow, A. (2018). Engaging Native American caregivers in youth-focused diabetes prevention and management. Preventing Chronic Disease, 15, Article 170521. https://doi.org/10.5888/pcd15.170521

DiMatteo, M. (2004). Social support and patient adherence to medical treatment: A meta-analysis. Health Psychology, 23, 207-218. https://doi.org/10.1037/0278 $-6133.23 .2 .207$

Fisher, L., Chesla, C., Mullan, J., Skaff, M., \& Kanter, R. (2001). Contributors to depression in Latino and European-American patients with type 2 diabetes. Diabetes Care, 24, 1751-1757. https://doi.org/10.2337/diacare.24.10.1751

Gallant, M. (2003). The influence of social support on chronic illness self-management: A review and directions for research. Health Education \& Behavior, 30, 170-195. https://doi.org/10.1177/1090198102251030

Garrett, M., Parrish, M., Williams, C., Grayshield, L., Portman, T., Rivera, E., \& Maynard, E. (2014). Invited commentary: Fostering resilience among Native American youth through therapeutic intervention. Journal of Youth and Adolescence, 43, 470-490. https://doi.org/10.1007/s10964-013-0020-8 
Goins, R., Noonan, C., Gonzales, K., Winchester, B., \& Bradley, V. L. (2017). Association of depressive symptomology and psychological trauma with diabetes control among older American Indian women: Does social support matter? Journal of Diabetes and Its Complications, 31, 669-674. https://doi.org/10.1016/j .jdiacomp.2017.01.004

Hill, D. (2006). Sense of belonging as connectedness, American Indian worldview, and mental health. Archives of Psychiatric Nursing, 20, 210-216. https://doi.org /10.1016/j.apnu.2006.04.003

Hu, L., \& Bentler, P. M. (1999). Cutoff criteria for fit indexes in covariance structure analysis: Conventional criteria versus new alternatives. Structural Equation Modeling: A Multidisciplinary Journal, 6, 1-55. https://doi.org/10.1080/10705519909540118

Idler, E. L., \& Benyamini, Y. (1997). Self-rated health and mortality: A review of twenty-seven community studies. Journal of Health and Social Behavior, 38, 21-37. https://doi.org/10.2307/2955359

Jiang, L., Beals, J., Whitesell, N., Roubideaux, Y., Manson, S., \& the AI-SUPERPFP Team. (2008). Stress burden and diabetes in two American Indian reservation communities. Diabetes Care, 31, 427-429. https://doi.org/10.2337/dc07-2044

Jones, D. J., \& Lindahl, K. M. (2011). Coparenting in extended kinship systems: African American, Hispanic, Asian heritage, and Native American families. In J.

P. McHale \& K. M. Lindahl (Eds.), Coparenting: A conceptual and clinical examination of family systems (pp. 61-79). Washington, DC: American Psychological Association.

Krieger, N. (2001). Theories for social epidemiology in the 21st century: An ecosocial perspective. International Journal of Epidemiology, 30, 668-677. https://doi .org/10.1093/ije/30.4.668

Krieger, N. (2014). Discrimination and health inequities. International Journal of Health Services, 44, 643-710. https://doi.org/10.2190/HS.44.4.b

Kulis, S., Hodge, D., Ayers, S., Brown, E., \& Marsiglia, F. (2012). Spirituality and religion: Intertwined protective factors for substance use among urban American Indian youth. The American Journal of Drug and Alcohol Abuse, 38, 444-449. https://doi.org/10.3109/00952990.2012.670338

LaFromboise, T. D., \& Dizon, M. (2003). American Indian children and adolescents. In J. Gibbs \& L. Huang (Eds.), Children of color: Psychological interventions with culturally diverse youth (pp. 45-90). San Francisco, CA: Jossey-Bass.

LaFromboise, T. D., Hoyt, D. R., Oliver, L., \& Whitbeck, L. B. (2006). Family, community, and school influences on resilience among American Indian adolescents in the upper Midwest. Journal of Community Psychology, 34, 193-209. https: //doi.org/10.1002/jcop.20090

Martin, D., \& Yurkovich, E. (2014). "Close-knit" defines a healthy Native American Indian family. Journal of Family Nursing, 20, 51-72. https://doi.org /10.1177/1074840713508604

Martin, D., Yurkovich, E., \& Anderson, K. (2016). American Indians' family health concern on a Northern plains reservation: "Diabetes runs rampant here." Public Health Nursing, 33, 73-81. https://doi.org/10.1111/phn.12225 
Mohatt, N., Fok, C., Burket, R., Henry, D., \& Allen, J. (2011). Assessment of awareness of connectedness as a culturally-based protective factor for Alaska native youth. Cultural Diversity \& Ethnic Minority Psychology, 17, 444-455. https: //doi.org/10.1037/a0025456

Muthén, L. K., \& Muthén, B. O. (2007). Mplus user's guide (5th ed.). Los Angeles, CA: Author.

Naranjo, D., Hessler, D., Deol, R., \& Chesla, C. (2012). Health and psychosocial outcomes in U.S. adult patients with diabetes from diverse ethnicities. Current Diabetes Report, 12, 729-738. https://doi.org/10.1007/s11892-012-0319-y

Nunally, J. (1978). Psychometric theory. New York, NY: McGraw-Hill.

Pearlin, L. I., Menaghan, E. G., Lieberman, M. A., \& Mullan, J. T. (1981). The stress process. Journal of Health and Social Behavior, 22, 337-356. https://doi .org/10.2307/2136676

Scarton, L., Bakas, T., Miller, W., Poe, G., \& Huber, L. (2014). Needs and concerns of family caregivers of persons with type 2 diabetes: An integrated review of cross-cultural literature with implications for the American Indian population. The Diabetes Educator, 40, 444-452. https://doi.org/10.1177/0145721714529831

Scarton, L., Bakas, T., Poe, G., Hull, M., Ongwela, L., \& Miller, W. (2016). Needs and concerns of family caregivers of American Indians, African Americans, and Caucasians with type 2 diabetes. Clinical Nursing Research, 25, 139-156. https: //doi.org/10.1177/1054773814562879

Schiefer, D., \& Krahé, B. (2014). Ethnic identity and orientation to White American culture are linked to well-being among American Indians-but in different ways. Social Psychology, 45, 1-14. https://doi.org/10.1027/1864-9335/a000155

Schnittker, J., \& Bacak, V. (2014). The increasing predictive validity of self-rated health. PLoS ONE, 9, e84933. https://doi.org/10.1371/journal.pone.0084933

Shields, C. G., Franks, P., Harp, J. J., Campbell, T. L., \& McDaniel, S. H. (1994). Family Emotional Involvement and Criticism Scale (FEICS): II. Reliability and validity studies. Family Systems Medicine, 12, 361-377. https://doi.org/10.1037 /h0089289

Sijtsma, K. (2009). Reliability beyond theory and into practice. Psychometrika, 74, 169-173. https://doi.org/10.1007/s11336-008-9103-y

Smart, S., St Germaine, E., \& Contributors. (1989). Lac du Flambeau AODA Parenting Program curriculum.

Szanton, S., Gill, J., \& Allen, J. (2005). Allostatic load: A mechanism of socioeconomic health disparities? Biological Research for Nursing, 7, 7-15. https://doi .org/10.1177/1099800405278216

Tashiro, C. (2005). Health disparities in the context of mixed race: Challenging the ideology of race. Advances in Nursing Science, 28, 7-15. https://doi.org /10.1097/00012272-200507000-00003

Tiedt, J. A., \& Brown, L. A. (2014). Allostatic load: The relationship between chronic stress and diabetes in Native Americans. Journal of Theory Construction \& Testing, 18, 22-27. Retrieved from https://search.proquest.com/docview/16398 41745 ?accountid $=32710$ 
van Dam, H., van der Horst, F., Knoops, L., Ryckman, R., Crebolder, H., \& van den Borne, B. (2005). Social support in diabetes: A systematic review of controlled intervention studies. Patient Education and Counseling, 59, 1-12. https://doi .org/10.1016/j.pec.2004.11.001

Walders-Abramson, N., Venditti, E. M., Ievers-Landis, C. E., Anderson, B., El Ghormli, L., Geffner, M., . . . Yasuda, P. (2014). Relationships among stressful life events and physiological markers, treatment adherence, and psychosocial functioning among youth with type 2 diabetes. The Journal of Pediatrics, 165 , 504-508. https://doi.org/10.1016/j.jpeds.2014.05.020

Walls, M., Johnson, K., Whitbeck, L., \& Hoyt, D. (2006). Mental health and substance abuse services references and utilization patterns among American Indian people of the Northern Midwest. Community Mental Health Journal, 42, 521535. https://doi.org/10.1007/s10597-006-9054-7

Walters, K., Simoni, J., \& Evans-Campbell, T. (2002). Substance use among American Indians and Alaska Natives: Incorporating culture in an "indigenist" stress-coping paradigm. Public Health Reports, 117, S104-S117.

Wittchen, H.-U., \& Kessler, R. C. (1994). Modifications of the CIDI in the National Comorbidity Study: The development of the UM-CIDI (NCS Working Paper \#2). Ann Arbor, MI. Retrieved from https:/www.hcp.med.harvard.edu/ncs/ftpdir/um -cidi.pdf

Xu, J., Murphy, S. L., Kochanek, K. D., \& Bastian, B. A. (2016). Deaths: Final data for 2013 (National Vital Statistics Reports, Vol. 64, No. 2). Hyattsville, MD: National Center for Health Statistics. Retrieved from https://www.cdc.gov/nchs /data/nvsr/nvsr64/nvsr64_02.pdf

\section{Author Biographies}

Ashleigh Coser, MS (Muscogee Creek, Choctaw, and Chickasaw Nations), is a doctoral candidate in the clinical psychology program at Oklahoma State University, Stillwater, Oklahoma, the United States. Her primary research interests include examining the parent-child relationship and involvement of extended family and tribal community among American Indian families. Her recent publications include "An Investigation of American Indian Parenting, Child Behavior, and Extended Family Involvement" (2014).

Kelley J. Sittner, $\mathrm{PhD}$, is an associate professor in the Department of Sociology at Oklahoma State University, Stillwater, Oklahoma, the United States. Her research focuses on physical and mental health, antisocial behavior, and substance use among Indigenous youth and adults. Her recent publications include "Microaggressions, Diabetes Distress, and Self-Care Behaviors in a Sample of American Indian Adults With Type 2 Diabetes" in Journal of Behavioral Medicine (2018, with B. L. Greenfield \& M. L. Walls) and "Stress Exposure and Physical, Mental, and Behavioral Health Among American Indian Adults With Type 2 Diabetes" in International Journal of Environmental Research and Public Health (2017, with M. L. Walls, B. D. Aronson, A. K. Forsberg, L. B. Whitbeck, \& M. al'Absi). 
Melissa L. Walls, PhD (Bois Forte and Couchiching First Nation Ojibwe), is an associate professor at the University of Minnesota Medical School, Duluth campus in Duluth, Minnesota, the United States. She works in collaboration with tribal communities to address the sociohistorical determinants of health inequities. Her recent publications include "Stress Exposure and Physical, Mental, and Behavioral Health Among American Indian Adults With Type 2 Diabetes" in International Journal of Environmental Research and Public Health (2017, with K. J. Sittner, B. D. Aronson, A. K. Forsberg, L. B. Whitbeck, \& M. al'Absi), "Examining Protective and Buffering Associations Between Sociocultural Factors and Adverse Childhood Experiences Among American Indian Adults With Type 2 Diabetes: A Quantitative, CommunityBased Participatory Research Approach" in BMJ Open (2018, with T. N. Brockie \& J. H. L. Elm), and "The Prevalence and Correlates of Mental and Emotional Health Among American Indian Adults With Type 2 Diabetes" in The Diabetes Educator (2014, with B. D. Aronson, G. V. Soper, \& M. D. Johnson-Jennings).

Tina Handeland, MS (Lac du Flambeau Ojibwe), is a member of the Maawaji' idioog Mino-ayaawin (Gathering for Health) Community Research Committee in Lac du Flambeau, Wisconsin, the United States. She has been working with children and families on the reservation relating to AODA, parenting programs, prevention activities, and intervention service. 